\title{
Massive Hemolysis Causing Renal Failure in Acute Hepatitis E Infection
}

\author{
Pragya Karki ${ }^{1}$, Sarthak Malik ${ }^{2}$, Bipadabhanjan Mallick ${ }^{2}$, Vishal Sharma*² and Surinder S Rana ${ }^{2}$ \\ ${ }^{1}$ Department of Internal Medicine, PGIMER, Chandigarh, India; ${ }^{2}$ Department of Gastroenterology, PGIMER, Chandigarh, India
}

\begin{abstract}
Acute viral hepatitis is usually a self-limiting illness. However, it can lead to complications that can be life-threatening, such as acute liver failure. Glucose 6 phosphate dehydrogenase (G6PD) deficiency in the setting of acute viral hepatitis can lead to a massive hemolysis, manifesting as acute kidney injury and markedly raised bilirubin levels; although cases are rare. Here, we report such a case. The patient had a viral hepatitis $E$ infection and presented with kidney injury requiring dialysis. Examination showed very high mixed hyperbilirubinemia due to massive intravascular hemolysis. The patient experienced a long, protracted course of illness, requiring renal replacement therapy with other supportive management, which led to improvement over a period of four weeks. This case highlights the importance of recognizing associated hemolysis in a patient with viral hepatitis who presents with very high bilirubin levels or associated kidney injury. Such patients will require aggressive supportive care with prompt fluid and electrolyte management.

(c) 2016 The Second Affiliated Hospital of Chongqing Medical University. Published by XIA \& HE Publishing Inc. All rights reserved.
\end{abstract}

\section{Introduction}

Hepatitis E virus (HEV) is an important cause of acute viral hepatitis in the developing world. Glucose- 6 phosphate dehydrogenase (G6PD) deficiency reportedly affects $2.2-14 \%$ of the general population in North India. ${ }^{1}$ Though HEV usually presents as a mild self-limiting illness, its association with G6PD deficiency may lead to exaggerated intravascular hemolysis, severe hyperbilirubinemia and acute renal failure.

\section{Case report}

A 48-year-old man presented to emergency services with complaint of a two-week period of yellowish discoloration of the eyes and urine. For four days prior to presentation, these

Keywords: Hepatitis E virus; Hemolysis; Anemia; Hemoglobinuria; G6PD deficiency.

Abbreviations: HEV, hepatitis E virus; G6PD, glucose 6 phosphate dehydrogenase; $\mathrm{LDH}$, lactate dehydrogenase; $\mathrm{KF}$, Kayser-Fleischer.

Received: 12 September 2016; Revised: 16 October 2016; Accepted: 08 November 2016

DOI: $10.14218 / \mathrm{JCTH} .2016 .00042$

*Correspondence to: Vishal Sharma, Department of Gastroenterology, PGIMER, Chandigarh 160012, India. Tel: +91-708-7008099, Fax: +91-172-2744401, E-mail: docvishalsharma@gmail.com symptoms had been accompanied by reduced urine output. One day prior to presentation, the patient developed slurring of speech with altered sleep-wake pattern. The patient had history of fever, which subsided with onset of jaundice; the fever was reportedly intermittent and not associated with chills or rigors, and which subsided by antipyretic. There was no history of any alcohol or drug intake, or of any previous neurological symptoms or family history of Wilson's disease.

On examination, the patient was conscious but disoriented, and in appearance was pale and deeply icteric. Abdominal examination revealed hepatomegaly at $3 \mathrm{~cm}$ below the right costal margin, with no splenomegaly. Shifting dullness was present. Neurological examination revealed asterixis. There was no focal deficit. Laboratory investigations revealed hemoglobin of $5.8 \mathrm{~g} / \mathrm{dL}$, total leucocyte count of $5700 / \mu \mathrm{L}$, platelet count of $320000 / \mu \mathrm{L}$, total bilirubin of $53.39 \mathrm{mg} / \mathrm{dL}$ (conjugated fraction of $20.25 \mathrm{mg} / \mathrm{dL}$ ), aspartate transaminase of $4553 \mathrm{U} / \mathrm{L}$, alanine aminotransferase of $3907 \mathrm{U} / \mathrm{L}$, lactate dehydrogenase (LDH) of $6230 \mathrm{U} / \mathrm{L}$, serum creatinine of 3.4, prothrombin time of $44 \mathrm{~s}$ (control: $12 \mathrm{~s}$ ) and international normalized ratio of 3.03. The workup for jaundice showed positivity for IgM antiHEV (EIAgen HEV IgM kit; Adaltis, Spain) and negativity for hepatitis A antibody IgM (IgM anti-HAV kit; Adaltis), hepatitis B surface antigen (HBsAg) (Monalisa HBsAg Plus kit; Bio-Rad, USA) and hepatitis C virus antibody (Innotest HCV ab IV kit; Innogenetics, Belgium). Serum ceruloplasmin levels were normal and autoimmune markers (antinuclear antibody, smooth muscle antibody, liver-kidney microsomal antibody) were negative. There was no Kayser-Fleischer (KF) ring.

In view of the markedly increased bilirubin and low hemoglobin, the possibility of intravascular hemolysis was kept in mind. On evaluation, the patient was found to be deficient for G6PD, with raised plasma hemoglobin levels. Direct and indirect Coombs tests were negative. Urine examination revealed hemoglobinuria. The patient's renal functions worsened, and creatinine gradually increased to $8.1 \mathrm{mg} / \mathrm{dL}$. Considering the coagulopathy, encephalopathy and intravascular hemolysis, a diagnosis of HEV-related acute liver failure with hemolysis with acute kidney injury was made. The patient was managed with hemodialysis, transfusion of packed red cells, intravenous antibiotics and other supportive measures, and a gradual improvement in the patient's status occurred over the next four weeks. Hemoglobin levels increased to $7.9 \mathrm{~g} / \mathrm{dL}$ and liver and renal functions normalized (Fig. 1). The patient was discharged in stable condition and remains well one month after the discharge. At the three-month follow-up, a repeat anti-HCV antibody test was negative. 

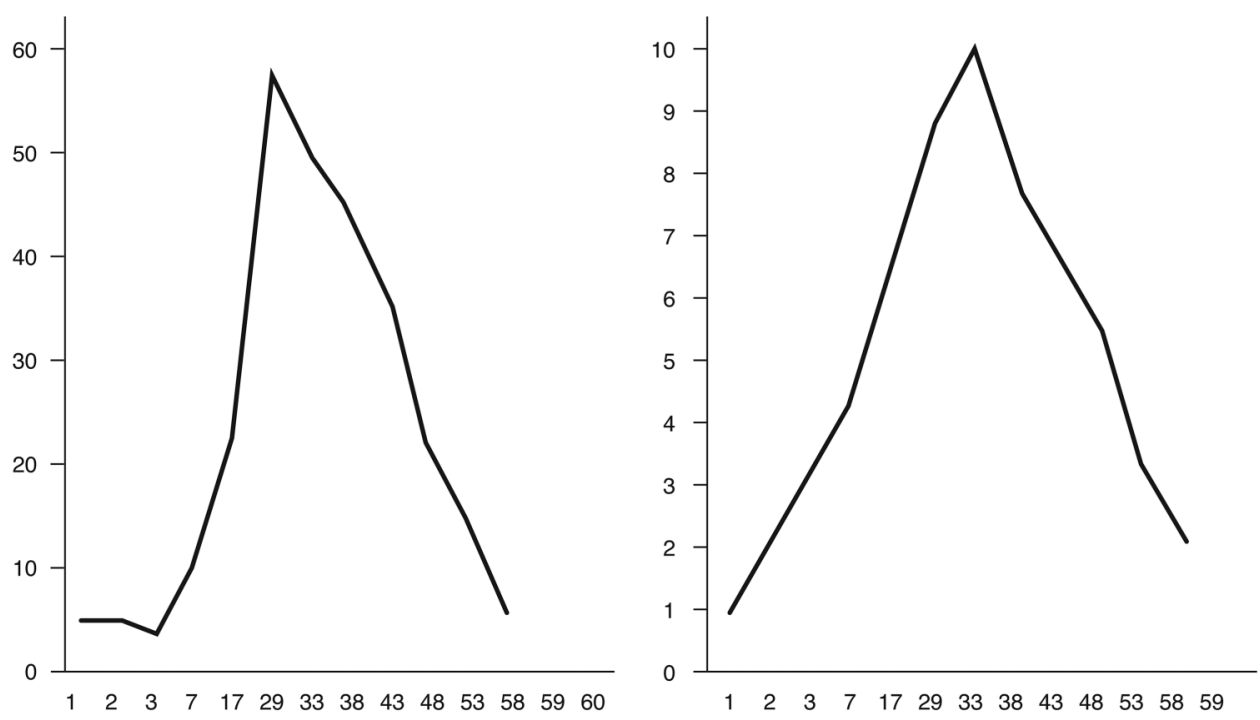

Duration of Illness(in days)

Fig. 1. Bilirubin and creatinine changes in the index patient.

\section{Discussion}

HEV is one of the most common causes of acute viral hepatitis in northern India and is usually a self-limiting illness. ${ }^{2}$ However, in rare cases, it has been associated with severe life-threatening complications such as acute hemolytic crisis leading to acute renal failure on the background of G6PD deficiency. G6PD deficiency reportedly affects $2.2-14 \%$ of the north Indian population, and co-existence of both illnesses, though rare, has been documented. ${ }^{2,3}$ Acute viral hepatitis and HEV per se can lead to mild hemolysis; however, cases of severe hemolysis with markedly raised bilirubin with acute kidney injury, as in our patient, are very rare..$^{3,4}$ Our patient had severe intravascular hemolysis, as evidenced by the rapid fall in hemoglobin, marked elevation in mixed hyperbilirubinemia, hemoglobinuria, and markedly raised plasma LDH. Abid et al. ${ }^{3}$ were the first to describe this rare life-threatening association in a series of five cases, four of which experienced renal failure leading to a long, protracted course of illness. Occasional reports have also suggested that HEV can cause massive hemolysis and renal injury in patients with G6PD deficiency. ${ }^{4,5}$

The mechanism of hemolysis in G6PD deficient patients is oxidative stress, which may be precipitated by drugs or infection. Acute viral hepatitis leads to hepatic dysfunction, further leading to accumulation of harmful oxidants that result in reduced glutathione levels. Glutathione levels are already low in red blood cells of G6PD deficient subjects, however, and the further reduction due to viral hepatitis precipitates severe hemolysis. ${ }^{6,7}$ A recent review of all such cases reported to date suggested that renal failure occurs in the setting of high bilirubin levels, but the exact cause remained undetermined. ${ }^{8}$ It has been proposed that acute tubular necrosis and mechanical tubular obstruction due to hematin and bilirubin may contribute. ${ }^{7}$ Wilson's disease can also present as an acute hemolytic crisis, however, it was excluded in our case according to the ceruloplasmin levels being normal and the KF ring being absent. Our patient developed acute renal failure, requiring three sessions of hemodialysis. During subsequent outpatient visits, testing of the viral markers (HBs antigen, IgM anti-HBc, HCV RNA and anti-HCV) was repeated and negative results obtained. We again repeated testing for ceruloplasmin levels and 24-hour urine copper level, and both were found to be normal. The possibility of Wilson's disease was very low, as Wilsonian acute liver failure doesn't improve short of liver transplant.

In light of the case presented herein, we suggest that physicians consider the possibility of hemolysis for patients with acute viral hepatitis and very high bilirubin levels. Testing of G6PD levels, even when results are normal, should (ideally) be repeated at 6-8 weeks, as levels can be falsely normal due to the presence of fresh red blood cells in the circulation following a hemolytic episode. A patient with hemolysis who develops renal failure should be managed carefully, with optimal correction of fluid and electrolyte imbalance, and avoidance of nephrotoxic and hemolysis-producing drugs.

\section{Conflict of interest}

None

\section{Author contributions}

Wrote the initial draft (PK), edited the article (VS, SSR), helped in literature search (SM, BM), participated in care of the patient (PK, SM, BM, VS, SSR). 
Karki P. et al: HEV-related massive hemolysis

\section{References}

[1] Das K, Agarwal A, Andrew R, Frösner GG, Kar P. Role of hepatitis E and other hepatotropic virus in aetiology of sporadic acute viral hepatitis: a hospital based study from urban Delhi. Eur J Epidemiol 2000;16:937-940. doi: 10. 1023/A: 1011072015127.

[2] Goyal M, Garg A, Goyal MB, Kumar S, Ramji S, Kapoor S. Newborn screening for G6PD deficiency: A 2-year data from North India. Indian J Public Health 2015;59:145-148. doi: 10.4103/0019-557X.157537.

[3] Abid S, Khan AH. Severe hemolysis and renal failure in glucose-6-phosphate dehydrogenase deficient patients with hepatitis E. Am J Gastroenterol 2002; 97:1544-1547. doi: 10.1111/j.1572-0241.2002.05740.x.

[4] Monga A, Makkar RP, Arora A, Mukhopadhyay S, Gupta AK. Case report: Acute hepatitis $E$ infection with coexistent glucose-6-phosphate dehydrogenase deficiency. Can J Infect Dis 2003;14:230-231. doi: 10.1155/2003/913679.
[5] Tomar LR, Aggarwal A, Jain P, Rajpal S, Agarwal MP. Acute viral hepatitis E presenting with haemolytic anaemia and acute renal failure in a patient with glucose-6-phosphate dehydrogenase deficiency. Trop Doct 2015;45:245-246. doi: 10.1177/0049475514559959.

[6] Arese P, De Flora A. Pathophysiology of hemolysis in glucose-6-phosphate dehydrogenase deficiency. Semin Hematol 1990;27:1-40.

[7] Agarwal RK, Moudgil A, Kishore K, Srivastava RN, Tandon RK. Acute viral hepatitis, intravascular haemolysis, severe hyperbilirubinaemia and renal failure in glucose-6-phosphate dehydrogenase deficient patients. Postgrad Med J 1985;61:971-975. doi: 10.1136/pgmj.61.721.971.

[8] Bazerbachi F, Haffar S, Garg SK, Lake JR. Extra-hepatic manifestations associated with hepatitis $E$ virus infection: a comprehensive review of the literature. Gastroenterol Rep (Oxf) 2016;4:1-15. doi: 10.1093/ gastro/gov042. 